\title{
Theoretical analysis and simulation study of the deep overcompression mode of velocity bunching for a comblike electron bunch train
}

\author{
Dan Wang, Lixin Yan, YingChao Du, Wenhui Huang, Wei Gai, and Chuanxiang Tang \\ Department of Engineering Physics, Tsinghua University, Beijing 100084, China
}

(Received 12 December 2017; published 27 February 2018)

\begin{abstract}
Premodulated comblike electron bunch trains are used in a wide range of research fields, such as for wakefield-based particle acceleration and tunable radiation sources. We propose an optimized compression scheme for bunch trains in which a traveling wave accelerator tube and a downstream drift segment are together used as a compressor. When the phase injected into the accelerator tube for the bunch train is set to $\ll-100^{\circ}$, velocity bunching occurs in a deep overcompression mode, which reverses the phase space and maintains a velocity difference within the injected beam, thereby giving rise to a compressed comblike electron bunch train after a few-meter-long drift segment; we call this the deep overcompression scheme. The main benefits of this scheme are the relatively large phase acceptance and the uniformity of compression for the bunch train. The comblike bunch train generated via this scheme is widely tunable: For the two-bunch case, the energy and time spacings can be continuously adjusted from +1 to $-1 \mathrm{MeV}$ and from 13 to $3 \mathrm{ps}$, respectively, by varying the injected phase of the bunch train from $-220^{\circ}$ to $-140^{\circ}$. Both theoretical analysis and beam dynamics simulations are presented to study the properties of the deep overcompression scheme.
\end{abstract}

DOI: $10.1103 /$ PhysRevAccelBeams.21.024403

\section{INTRODUCTION}

The application ranges of free electron lasers (FELs), wakefield-based accelerators, and tunable radiation sources can be greatly extended by driving them with a train of short electron microbunches rather than with a single bunch [1-9]. For example, a bunch train in which the energy increases or decreases along its length can produce a corresponding train of radiation pulses of variable delays and wavelengths, which has potential applications in ultrafast pump-probe FEL experiments [8,10-18]. In addition, a bunch train with a subpicosecond bunch length and a repetition rate of a few terahertz can be quite useful in the coherent excitation of plasma waves in plasma wakefield accelerators $[19,20]$ and in the generation of frequencytunable narrow-band terahertz radiation $[7,21,22]$ as well as in other beam dynamics applications [5,23-25]. All of these challenges and developments have generated considerable interest in electron bunch trains, in which two or more electron bunches are generated and are then accelerated and compressed in the same accelerating bucket.

\footnotetext{
*wangdanzei@gmail.com
}

Published by the American Physical Society under the terms of the Creative Commons Attribution 4.0 International license. Further distribution of this work must maintain attribution to the author(s) and the published article's title, journal citation, and DOI.
There are several typical methods of generating such bunch trains, including the conversion of a transverse modulation into a periodic longitudinal distribution through the use of a dispersive beam line and an initial energy chirp [3] as well as the emittance-exchange technique [26,27]. However, the generation of a transverse modulation using a mask will always lead to particle loss. Another possibility is to introduce an energy modulation by means of self-excited wakefields and then convert it into a density modulation through a magnetic chicane [28]. However, the most straightforward method of generating microbunches is to impose modulations on the beam directly at the cathode and then attempt to maintain or recover these initial modulations as the beam propagates in the beam line [2,6-8].

The well-known "laser-comb" operating concept, in which terahertz bunch trains are generated by illuminating a photocathode with trains of laser pulses, was proposed by Boscolo et al. [29]. With this approach, it is possible to generate bunch trains with a charge of several hundreds of picocoulombs within the same rf gun accelerating bucket. Downstream of the gun exit, a velocity bunching technique is applied within a traveling wave accelerator (TWA) tube to modulate the bunch train by compressing each subbunch and adjusting the time and energy spacings along the train [2,29-31]. Such comblike bunch trains have been successfully used in X-ray FEL experiments $[12,14,17]$. However, from previously reported beam dynamics results [31-33], in which the velocity bunching of the bunch train mainly occurred when the phase injected into the TWA tube was in 


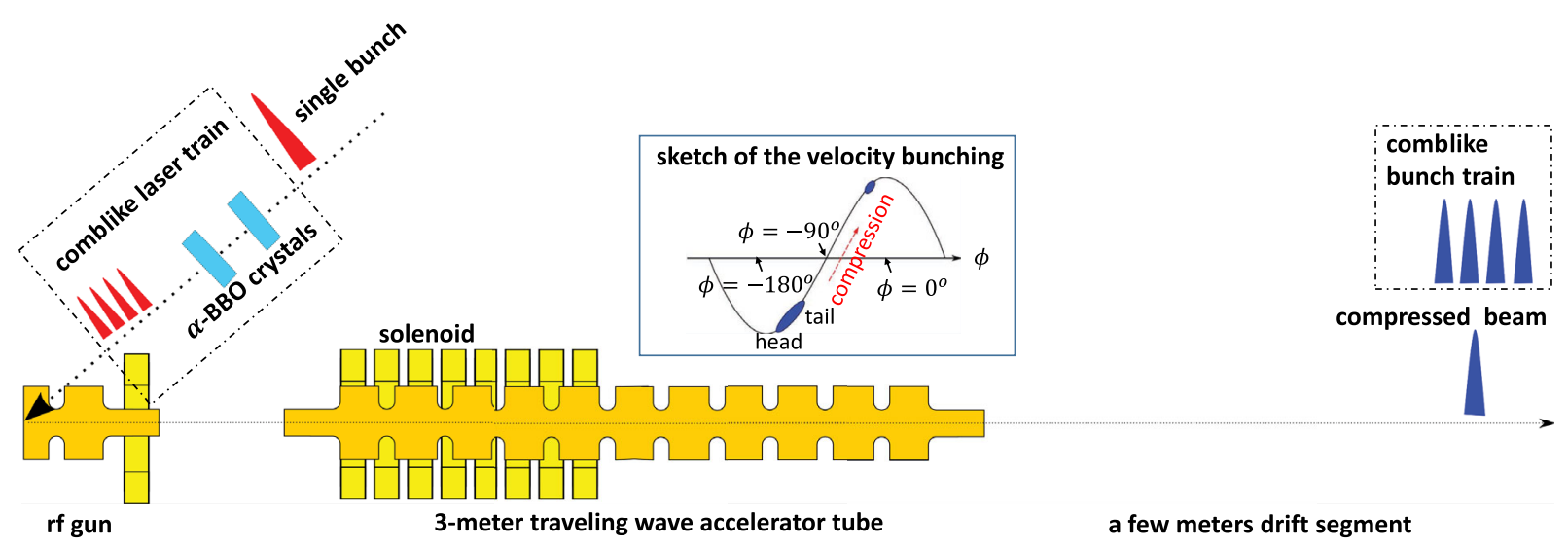

FIG. 1. Sketch of the velocity bunching scheme at the TTX beam line.

the range of $\sim[-100,-80]^{\circ}$, it is evident that this method suffers from a smearing effect from longitudinal space charge forces, which causes the initial modulation to be blurred and tend to disappear for high beam currents.

Here, we investigate the optimization of the velocity bunching of a bunch train generated via the laser-comb method under a different working condition; we call it as the deep overcompression scheme for short. This scheme was developed at the Tsinghua Thomson X-ray (TTX) beam line [34] and is illustrated in Fig. 1. With a TWA tube and a downstream drift segment together serving as a compressor, we have studied the injection of a very wide range of phases into the TWA tube for the first time. From both theoretical predictions and beam dynamics simulations, we find that, when the phase of the TWA tube is varied over a large range of values of $\ll-100^{\circ}$, the generated laser-comb bunch train is widely tunable in both energy and bunch interval after a few meters of drift. In this scheme, the TWA tube overcompresses the beam, reversing the phase space of the bunch train while maintaining a velocity difference (or energy chirp) within the bunch train at the TWA exit; then, the long drift segment gives rise to gentle and uniform compression of the bunch train. The main benefits of the deep overcompression scheme compared with other velocity bunching schemes are (i) the relatively large phase acceptance of the TWA tube and (ii) the uniformity of the compression for each subbunch in the laser-comb train.

This paper is organized as follows: First, a theoretical model without a space charge effect is built to study the deep overcompression scheme, and simulations of the compression of a single bunch with and without a space charge effect are also presented for comparison with the theory. Next, beam dynamics simulations with a space charge effect performed using ASTRA [35] are reported for the two-bunch case, in which we show that the two compressed bunches are relatively uniform under deep overcompression and that the time and energy spacings of the two bunches are continuously adjustable over a wide range. Finally, the key results for four bunches under deep overcompression are presented for both the low-charge case and the high-charge case; these results demonstrate the applicability of the optimized compression scheme for generating comblike beams.

\section{THEORY OF VELOCITY BUNCHING FOR A SINGLE BUNCH}

The velocity bunching scheme, also called rf compression, was previously investigated in Refs. [36,37]. The key principle of velocity bunching is that the compression and acceleration of the beam occur simultaneously within the same linac section.

Here, we concentrate on optimizing the velocity bunching scheme for more uniform compression of the bunch train, considering the effect of drift downstream of the TWA tube and performing a very wide scan of the phase injected into the TWA tube. We take the TTX beam line setup as an example, as shown in Fig. 1. A single laser bunch (or a comblike laser train generated with $\alpha$ - $\beta$-barium borate crystals) is generated and illuminated on the photocathode to produce an electron beam (or bunch train), which is then accelerated by a 1.6 cell $S$-band rf gun with a high gradient to control the space charge effect. The 3 -meter-long $S$-band TWA tube is placed $\sim 1.5 \mathrm{~m}$ away from the gun, and there is a few-meter-long downstream drift segment after the TWA tube.

In the TTX beam line, the TWA tube and the downstream drift segment together act as a compressor. In this section, we elaborate on a simple model for single-bunch compression without a space charge effect that describes how the drift segment affects the velocity bunching scheme.

In an rf TWA structure, the electrons experience a longitudinal electric field, and, thus, the longitudinal phase space obeys the following expressions:

$d \gamma / d z=-\alpha k \sin \phi, \quad d \phi / d z=k\left[1-\gamma / \sqrt{\left(\gamma^{2}-1\right)}\right]$,

where $d \gamma / d z$ and $d \phi / d z$, respectively, describe the changes in the Lorentz factor (energy) and the electron phase respect to the rf wave. 
Here, $\alpha=e E_{\text {peak }} / m_{0} c^{2}$, where $E_{\text {peak }}$ is the peak field of TWA, $k$ is the rf wave number, and $e, m_{0}$, and $c$ are constants representing the electron charge, the electron mass, and the speed of light, respectively.

In the drift segment, where $\alpha=0$,

$$
d \gamma / d z=0, \quad d \phi^{*} / d z=k\left[1-\gamma_{e} / \sqrt{\left(\gamma_{e}^{2}-1\right)}\right] .
$$

The symbol $\phi^{*}$ denotes the phase after the drift segment. The second expression in Eq. (2) can be simplified to

$$
\phi^{*}=\phi_{e}+\Delta \phi
$$

where $\phi_{e}$ is the phase at the exit of the TWA tube and $\Delta \phi$ is the phase change induced by the drift segment:

$$
\Delta \phi=k L\left[1-\gamma_{e} / \sqrt{\left(\gamma_{e}^{2}-1\right)}\right] \approx k L / 2 \gamma_{e}^{2} .
$$

Here, $L$ is the length of the drift segment after the TWA tube, and $\gamma_{e}$ is the Lorentz factor for electrons at the exit of the TWA tube, which remains unchanged in the drift segment. Equation (4) shows that $\Delta \phi$ is larger when $\gamma_{e}$ is smaller.

For an electron with an initial state $\left(\gamma_{0}, \phi_{0}\right)$, where $\gamma_{0}$ and $\phi_{0}$ denote the injected energy and phase, respectively, at the entrance of the TWA tube, Eq. (1) can be numerically solved using the Runge-Kutta method; thus, we can obtain the electron state $\left(\gamma_{e}, \phi_{e}\right)$ at the exit of the TWA tube. By combining the results with Eqs. (4) and (3), we can determine $\Delta \phi$ and $\phi^{*}$, respectively. We solve Eqs. (1)-(4) with the beam line setup depicted in Fig. 1, and the operation parameters are listed in Table I. The rf gun is set to a high gradient and the maximum acceleration phase $\left(0^{\circ}\right)$ to control the upstream space charge effect; thus, the initial state of the beam injected into the TWA tube is defined. We conduct a scan over the possible values of the phase injected into the TWA tube, $\phi_{0}$, to optimize the compression scheme. In this particular case, the gradient of the TWA tube is set to $8 \mathrm{MV} / \mathrm{m}$, which results in a relatively small $\gamma_{e}$ for a large $\Delta \phi$.

The curve of $\phi_{e}$ versus $\phi_{0}$ is shown as the blue dashed curve in Fig. 2(a); it is a smooth curve with a single valley (point $A$ ). The curves of $\Delta \phi$ versus $\phi_{0}$ and $\phi^{*}$ versus $\phi_{0}$ are also shown in Fig. 2(a) as the black and red curves,

TABLE I. Parameters of the TTX beam line setup.

\begin{tabular}{lc}
\hline \hline Parameter & Value \\
\hline Gradient of the rf gun & $120 \mathrm{MV} / \mathrm{m}$ \\
Phase of the rf gun & $0^{\circ}$ \\
Energy of the beam at the gun exit & $5.5 \mathrm{MeV}$ \\
Phase injected into the TWA tube & $\phi_{0}$ \\
Gradient of the TWA tube & $8 \mathrm{MV} / \mathrm{m}$ \\
Length of the downstream drift segment & $6 \mathrm{~m}$ \\
\hline \hline
\end{tabular}

respectively. We can see that the addition of the phase induced by the drift segment downstream of the TWA tube $(\Delta \phi)$ to the phase at the exit of the TWA tube $\left(\phi_{e}\right)$ leads to a double-valley structure (points $B$ and $C$ ) in the final phase curve $\phi^{*}$.

For an electron beam, the injected phase $\phi_{0}$ is defined as the phase injected into the accelerating field for a reference particle in the beam that is located at the average position of the beam. We let $\sigma_{\phi_{0}}$ denote the initial rms phase (or rms bunch length) distribution of the beam at the entrance of the TWA tube and define the compression factor as follows:

$$
C=\sigma_{\phi^{*}} / \sigma_{\phi_{0}}
$$

Then, we can obtain the compression factor curves of $C$ versus $\phi_{0}$ for an electron beam with $\sigma_{\phi_{0}}=1^{\circ}$ from the curves in Fig. 2(a); the results are shown in Fig. 2(b). Peaks appear in the compression factor curves at points $A^{\prime}, B^{\prime}$, and $C^{\prime}$, because the $\phi_{e}$ and $\phi^{*}$ values of every particle in the beam tend to be the same at these points. Figure 2(b) shows that, at the exit of the TWA tube, the maximum compression occurs for $\phi_{0} \sim-90^{\circ}$ (point $A^{\prime}$ ), whereas after the long drift segment, two compression peaks appear at $\phi_{0}>-90^{\circ}$ (point $B^{\prime}$ ) and $\phi_{0}<-90^{\circ}$ (point $C^{\prime}$ ).

In the more general case, the drift length $L$ and the accelerating field $E$ in the TWA tube can also be tuned in the deep overcompression scheme. The compression factor
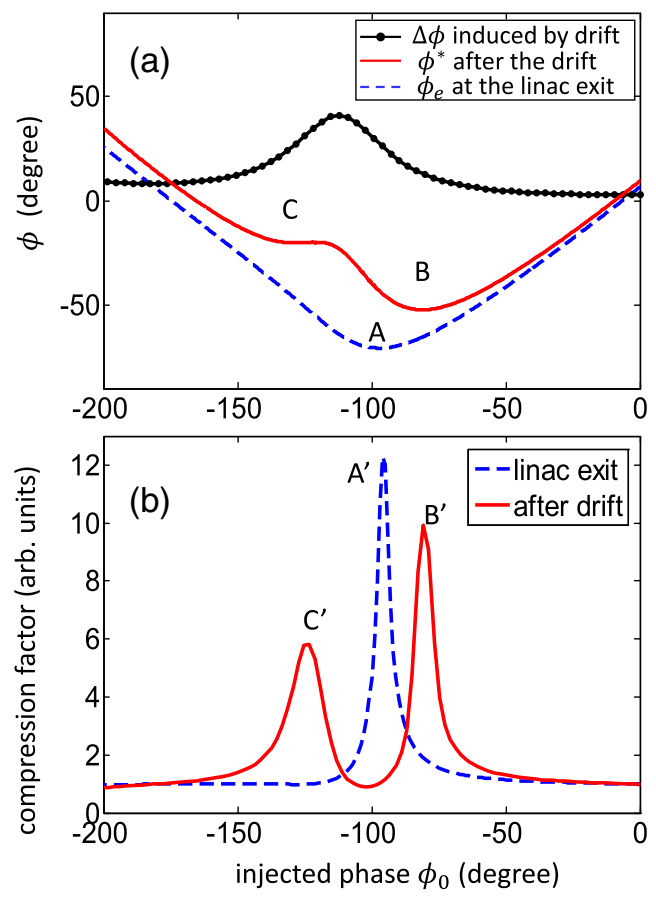

FIG. 2. Curves obtained by solving Eqs. (1)-(4) with the beam line parameters in Table I: (a) electron-phase-related quantities $\phi_{e}, \Delta \phi$, and $\phi^{*}$ versus the injected phase $\phi_{0}$ and (b) compression factor of the electron beam versus the injected phase $\phi_{0}$; the rms bunch length of the beam is $\sigma_{\phi_{0}}=1^{\circ}$. 

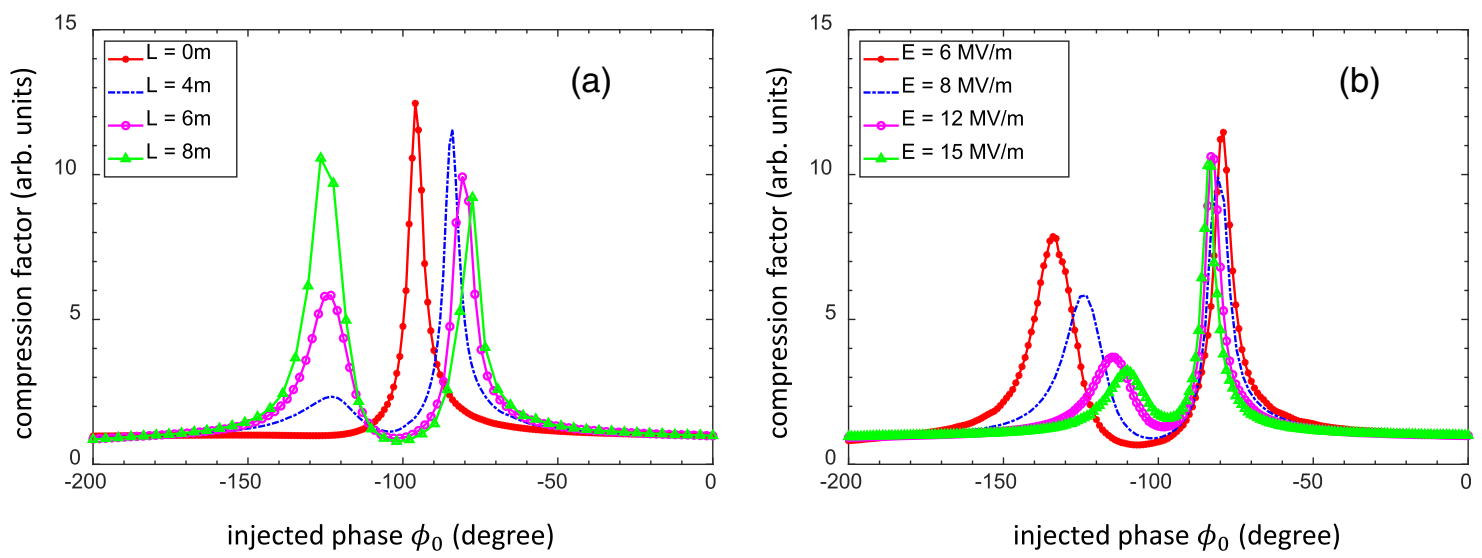

FIG. 3. Effects on the compression factor (a) when the gradient of the TWA tube is fixed at $8 \mathrm{MV} / \mathrm{m}$ and the drift length is varied and (b) when the drift length is fixed at $6 \mathrm{~m}$ and the gradient of the TWA tube is varied.

curves corresponding to different drift lengths when the gradient of the TWA tube is fixed at $8 \mathrm{MV} / \mathrm{m}$ are shown in Fig. 3(a), and the curves corresponding to different gradients of the TWA tube when the drift length is fixed at $6 \mathrm{~m}$ are shown in Fig. 3(b). The deep overcompression scheme benefits from a longer drift length and a lower gradient in the TWA tube, as the theory predicts. To strike a balance between the cost (which is higher for a longer beam line) and the energy of the beam at the target position, values of $L=6 \mathrm{~m}$ and $E=8 \mathrm{MV} / \mathrm{m}$ have been chosen for the TTX beam line; these values are used throughout the following discussions.

Velocity bunching can occur in several different compression modes depending on the phase $\phi_{0}$ injected into the TWA tube, as defined in Refs. [31,38]. As shown in Fig. 4, the different compression modes induced by different injected phases correspond to different variations in the rms bunch length along the beam line (position $Z$ ); the results are distinguished by differently colored curves (no space charge effect is included here). In all cases, the initial injected rms bunch length before compression is $1 \mathrm{ps}$ (black dashed line). In the full compression mode, corresponding to an injected phase of $\sim-90^{\circ}$ (magenta curve), the bunch length decreases to its minimum value at the accelerator exit. With an injected phase of $>-90^{\circ}$ (say, $-80^{\circ}$ ), the compression is inadequate, and some energy chirp remains in the beam at the exit of the tube, causing the minimum rms bunch length to be observed after $3 \mathrm{~m}$ of drift (blue curve). In the overcompression mode, corresponding to an injected phase of $<-90^{\circ}$ (say, $-100^{\circ}$ ), the rms bunch length monotonically increases after the TWA tube, and the minimum rms bunch length value occurs inside the TWA tube (green curve). By contrast, when the injected phase satisfies $\ll-90^{\circ}$ (say, $\sim-130^{\circ}$ ), there are two minima in the rms bunch length: One occurs inside the TWA tube, as in the overcompression mode, and the other occurs after a long drift of $6 \mathrm{~m}$ downstream from the TWA tube, as the red curve shows.
Now, let us consider the changes in the beam's phase space (distribution in $E-\phi$ space) in the deep overcompression scheme. We have marked points $A-G$ on the red curve in Fig. 4, and Fig. 5 shows the corresponding phase space at each of these points. The initial injected beam is a 2D Gaussian beam with an average energy of $5.5 \mathrm{MeV}$, as shown in Fig. 5 for point $A$. We have marked regions 1 and 2 of the beam to distinguish the time distributions. Since the phase injected into the TWA tube satisfies $\phi_{0} \ll-90^{\circ}$, the decelerated beam at point $B$ shows a chirp. Region 1 becomes slower than region 2, because it is subjected to more deceleration. Then, region 1 catches up with region 2 at point $C$, corresponding to the first occurrence of the minimum bunch length (inside the TWA tube). Because the beam is still inside the rf bucket, the beam continues to slip in phase and is subsequently accelerated, with an energy chirp again, as shown at point $D$. At point $E$, region 2 has surpassed region 1 such that the

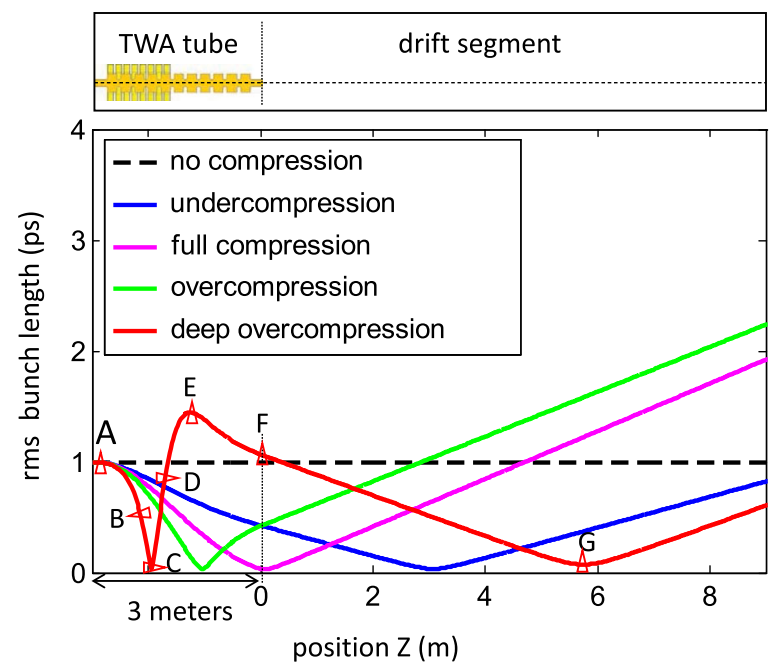

FIG. 4. Changes in rms bunch length along the beam line (position $Z$ ) for different compression modes. 


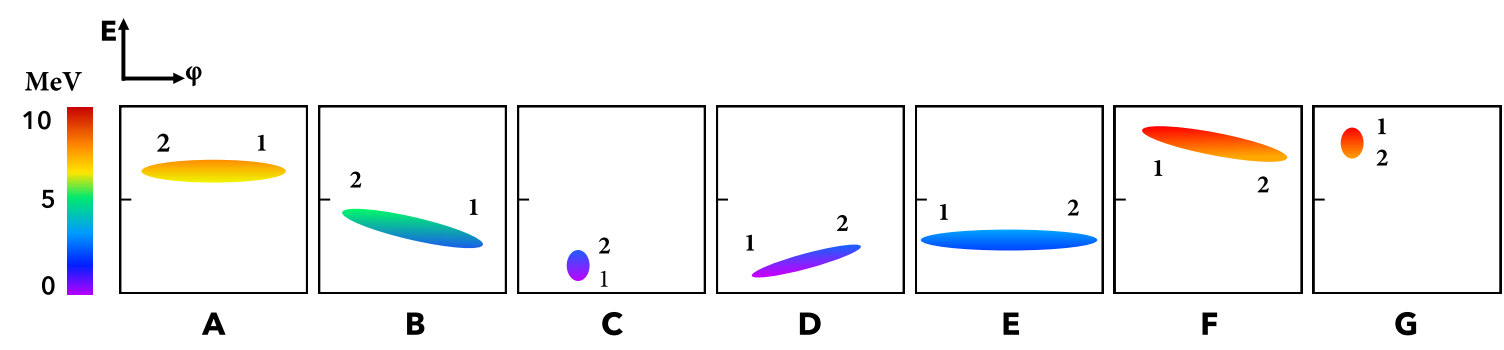

FIG. 5. Sketches of the changes in the electron beam phase space $(E-\phi)$ in the deep overcompression scheme at different positions along the beam line, where positions $A-G$ correspond to points $A-G$ on the red curve in Fig. 4.

bunch length reaches its maximum value inside the tube. Subsequently, the rf bucket continues accelerating and chirping the entire beam; at point $F$ (exit of the TWA tube), region 1 still has a higher energy than region 2, which results in another occurrence of the minimum bunch length after $6 \mathrm{~m}$ of drift, as shown for point $G$. It is clear that the energy space of the beam at point $G$ is reversed compared with that at point $C$. A long drift segment is necessary to obtain a compressed beam outside the tube in this scheme.

Up to this point, we have built a simple theoretical model without a space charge effect to explain the deep overcompression scheme. The results of beam dynamics simulations with and without a space charge effect performed using ASTRA code are also presented for comparison. The beam line setup and the detailed parameters used in the simulations are shown in Fig. 1 and Table I, and the results are shown in Fig. 6. For the velocity bunching scheme, we define the beam at the entrance of the TWA tube as the initial beam, which is traced from the laser with a space charge effect by means of the ASTRA code.

In Fig. 6, the beam's charge is $100 \mathrm{pC}$, and the initial bunch length is $\phi_{0}=1 \mathrm{ps}$. The compression factor is calculated as $\sigma_{f} / \sigma_{0}$, where $\sigma_{f}$ is the final rms bunch length after $6 \mathrm{~m}$ of drift downstream from the TWA tube. Two peaks are clearly seen in the compression factor curves for both cases (with and without a space charge effect), showing good agreement with the prediction represented by the red curve in Fig. 2(b). The green curve in Fig. 6(a), without a space charge effect, is more consistent with the theoretical prediction; the wide peak between the injected phase of $-150^{\circ}$ and $-110^{\circ}$ corresponds to the deep overcompression mode discussed above. From a comparison with the blue curve, we find that the complicated space charge effect that occurs when the beam is rotated during compression tends to increase both the height and the width of the peak in the compression factor curve that corresponds to the deep overcompression mode. By combining these findings with the behavior of the average energy curve (red curve), we find that the compression is stronger when the beam energy is relatively low; this finding is also consistent with Eq. (4). Figure 6(b) shows the simulated beam emittance versus $\phi_{0}$. The emittance is increased by up to a factor of 2 in the deep overcompression mode compared with the initial emittance when $\phi_{0}=0$.
Notably, the compression factor curve with the space charge effect [blue curve in Fig. 6(a)] is relatively flat and smooth in the deep overcompression range compared with the sharp peak at $\phi_{0} \sim-80^{\circ}$. This observation indicates that the deep overcompression mode has two advantages: (a) There is no abrupt change in the compression factor, as the injected phase $\phi_{0}$ is varied over a relatively large range; (b) the compression is more uniform across all subbunches when the separation or distribution (i.e., the occupied range in the phase space) of the injected bunches is relatively large. Because of these beneficial characteristics, the deep overcompression mode is broadly applicable to bunch trains with a wide variety of characteristics.
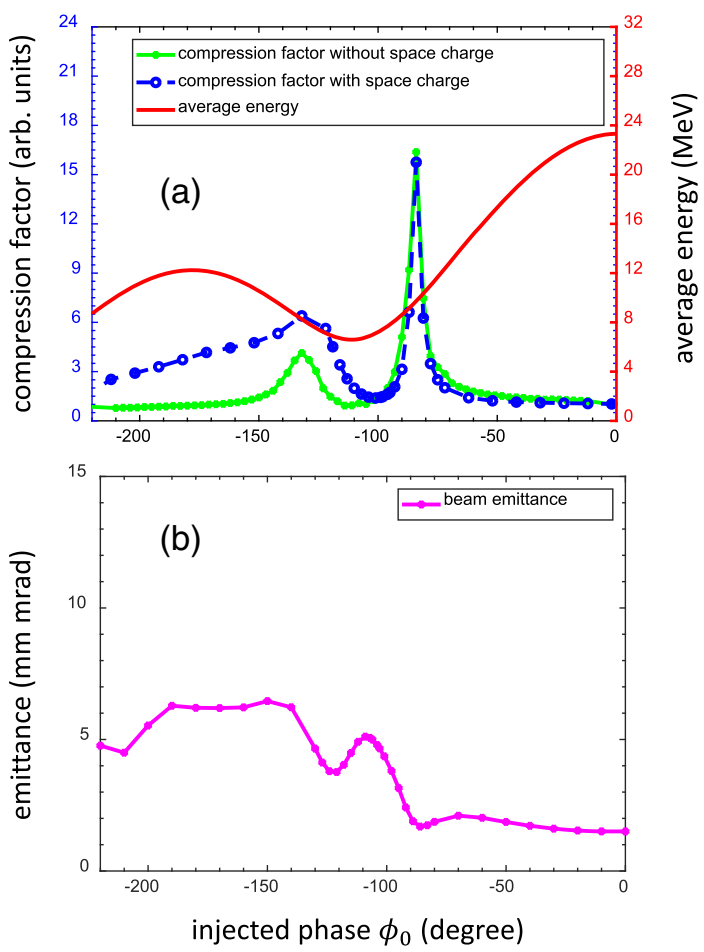

FIG. 6. Simulation results for single-bunch compression: (a) compression factors (with and without a space charge effect) and average beam energy versus the injected phase $\phi_{0}$ and (b) beam emittance versus the injected phase $\phi_{0}$. 


\section{TWO-BUNCH SIMULATIONS WITH A SPACE CHARGE EFFECT}

Typical simulation results for two-bunch compression with a space charge effect are shown in Fig. 7. The initial state of each subbunch with a charge of $100 \mathrm{pC}$ injected into the TWA tube is similar to that of the single bunch in Fig. 6. We use the colors red and blue to represent bunches 1 and 2, respectively; bunch 1 is in front of bunch 2 at injection. Not only the rms bunch length of each subbunch
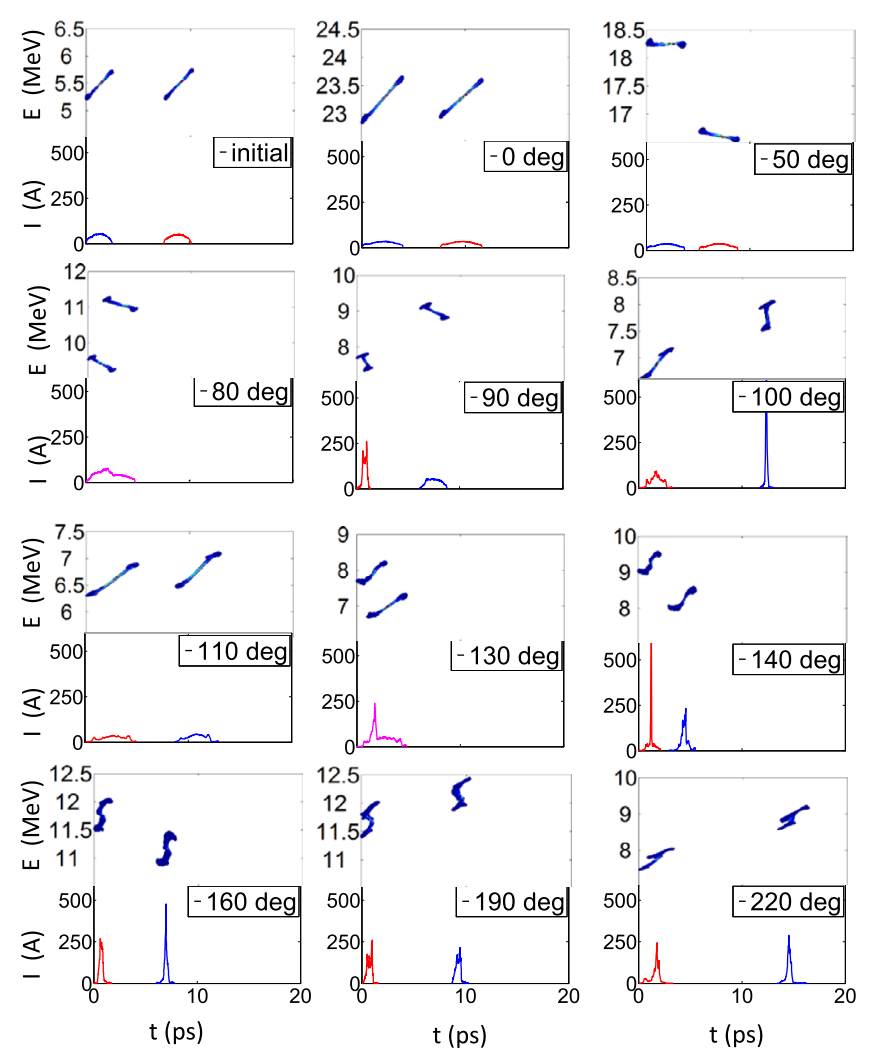

FIG. 7. ASTRA simulations of the longitudinal distributions and phase spaces for two-bunch compression with different injected phases $\phi_{0}$. but also the bunch interval vary with the injected phase $\phi_{0}$. Two special cases $\left(\phi_{0}=-80^{\circ}\right.$ and $\left.\phi_{0}=-130^{\circ}\right)$ are seen in the figure in which only one bunch (shown in magenta) appears, meaning that bunches 1 and 2 are merged together; these cases correspond to the peaks in the compression factor curve in Fig. 6 . We find that $\phi_{0}=-80^{\circ}$ is a dividing point that marks the reversal of bunches 1 and 2 in the time space; i.e., bunch 1 remains in front in the undercompression mode $\left(\phi_{0}>-80^{\circ}\right)$ but is behind bunch 2 when $\phi_{0}<-80^{\circ}$.

Figure 8 shows more details on two-bunch compression, including the bunch length and bunch emittance of each subbunch. As shown in Fig. 8(a), the bunch lengths of both bunches are compressed to relatively low values, and the lengths of the two bunches are more similar to each other in the deep overcompression mode (when $\phi_{0}$ is in the range of $\left.\left[-210^{\circ} \sim 150^{\circ}\right]\right)$, demonstrating the compression uniformity of the deep overcompression scheme. The bunch emittances of the subbunches are shown in Fig. 8(b); they exhibit similar trends as in the single-bunch case [magenta curve in Fig. 6(b)] but are obviously affected by the space charge effect between the bunches in the deep overcompression mode.

Figure 8(c) shows the bunch intervals with and without the space charge effect as well as the energy spacing versus the injected phase $\phi_{0}$. The initial interval and all intervals in the undercompression mode have positive values, indicating that bunch 1 is in front of bunch 2, whereas negative values indicate the reverse case, with bunch 2 in front. The two regions of zero values in the bunch interval curves correspond to the merged case $\left(\phi_{0}=-70^{\circ},-80^{\circ}\right.$ and $\phi_{0}=-120^{\circ},-130^{\circ}$ ). The accompanying values of the energy spacing between the two bunches have similar interpretations; i.e., positive values indicate that the average energy of bunch 1 is higher than that of bunch 2, whereas negative values indicate the opposite. In contrast to the marked space charge effect on the single-bunch compression curve [Fig. 6(a)], here the space charge effect on the bunch interval is slight, mainly because the charge of each subbunch is not very high $(100 \mathrm{pC})$ and the interval between the bunches is relatively large (a few picoseconds).
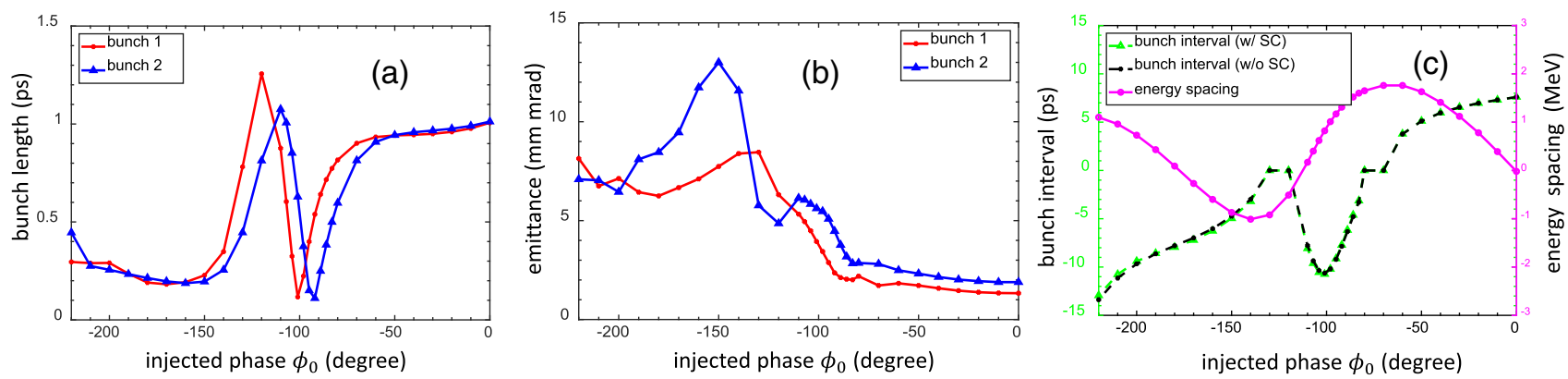

FIG. 8. Two-bunch compression results: (a) variation of the bunch length of each subbunch with $\phi_{0}$, (b) variation of the bunch emittance of each subbunch with $\phi_{0}$, and (c) variation of the bunch interval (with and without a space charge effect) and the energy spacing with $\phi_{0}$. 
We focus on the regions $\phi_{0}=-220^{\circ}$ to $-140^{\circ}$, where both the time and energy spacings are continuously adjustable over quite a large range $(+1$ to $-1 \mathrm{MeV}$ for the energy spacing between the two bunches and $13-3$ ps for the time spacing). Those results indicate the optimization of the bunch train tunability under the deep overcompression scheme.

\section{APPLICATION TO THE CASE OF A FOUR-BUNCH TRAIN}

The deep overcompression scheme can also be applied to improve the compression of a four-bunch train. We investigated the results in two cases: a low-charge bunch train, with a charge of $20 \mathrm{pC}$ in each subbunch, and a high-charge bunch train, with a charge of $200 \mathrm{pC}$ in each subbunch. The former case helps us to understand the compression uniformity of the deep overcompression scheme, and the latter case is more practical in application.

Figure 9 shows the low-charge case, in which the charge of each subbunch is $20 \mathrm{pC}$. The initial four-bunch train is generated by a four-bunch uniform laser with an interval of 3 ps upstream of the beam line in Fig. 1. It becomes nonuniform at the entrance to the TWA tube due to the
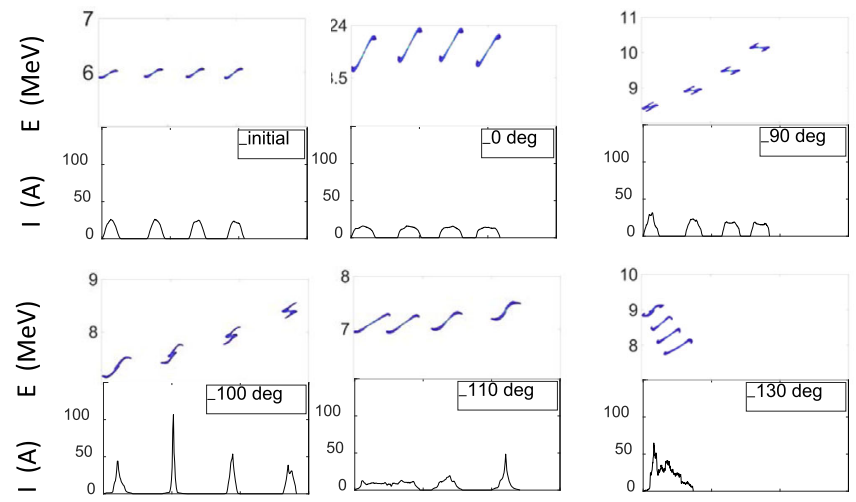

10
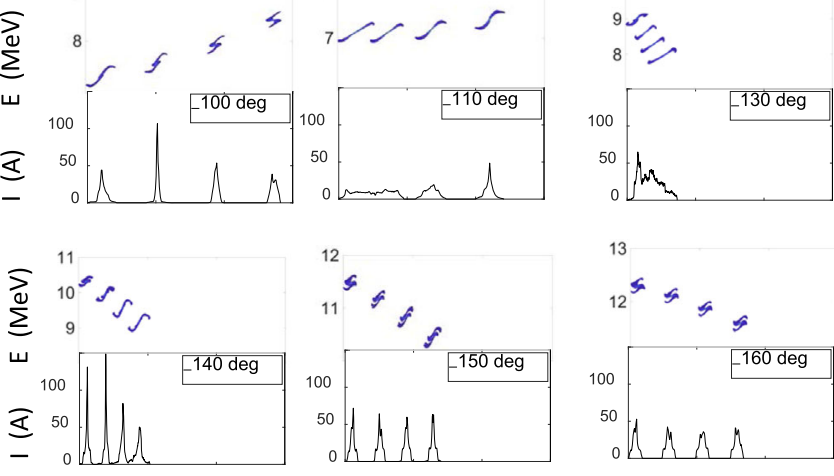

13
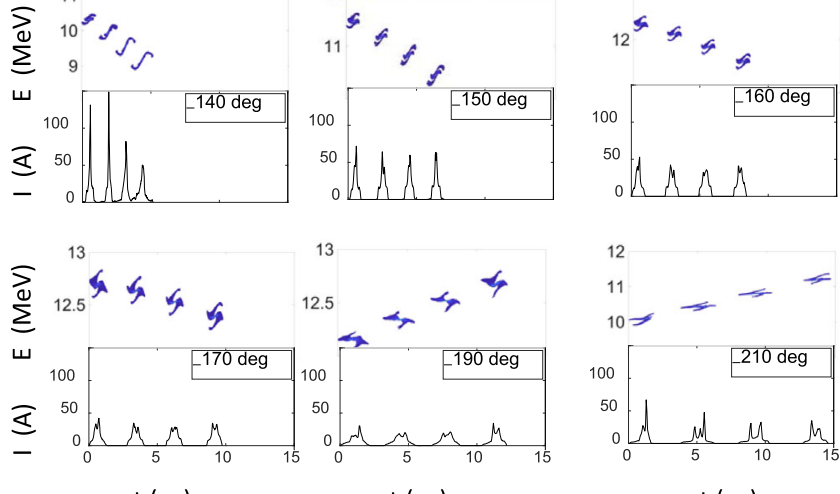

$t$ (ps)

$t$ (ps)

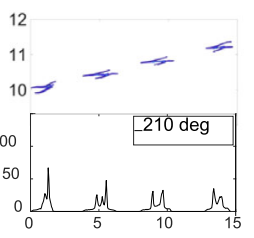

$t$ (ps)

FIG. 9. Low-charge four-bunch case: ASTRA simulations of the longitudinal distributions and phase spaces for the compression of a four-bunch train with different injected phases $\phi_{0}$, where the charge of each subbunch is $20 \mathrm{pC}$. energy chirp induced in the rf gun and the space charge effect. Then, the longitudinal distribution and phase space of the four-bunch train vary depending on the injected phase $\phi_{0}$ into the TWA tube. Our results mostly reproduce those previously reported in Refs. [32,33] for the case in which the injected phase satisfies $\phi_{0}>-110^{\circ}$, as shown in the top two rows in Fig. 9. However, with our understanding of the deep overcompression mode corresponding to $\phi_{0}<-130^{\circ}$, we obtain the typical optimized four-bunch train distributions shown in the bottom two rows in Fig. 9. For the case of $\phi_{0}=-150^{\circ}$, we achieve a relatively uniform distribution of the four-bunch train in the time space, with a current of $\sim 50 \mathrm{~A}$ for each compressed subbunch and a decreasing energy distribution. The average interval between adjacent subbunches is $\sim 1 \mathrm{ps}$. The four-bunch train distribution remains relatively uniformly spaced in time even when $\phi_{0}=-210^{\circ}$, for which the average interval between adjacent bunches is $\sim 5$ ps and their energy distribution is increasing.

To provide a closer look at the compression properties in the four-bunch case, we list detail parameters in Tables II [Fig. 10(a)] and III [Fig. 10(b)], which list the rms bunch lengths of each subbunch and the bunch intervals, respectively, for $4 \times 20 \mathrm{pC}$ bunch train with different $\phi_{0}$ values. And note that we list the results only when four bunches are separated as the value of $\phi_{0}$ is changed.

Figure 10 presents a summary of the parameters in the tables. The bunch lengths [Fig. 10(a)] and the bunch intervals [Fig. 10(b)] are tunable in the deep overcompression scheme. Moreover, the error bars show the standard deviations of the values, which in the deep overcompression mode are smaller than those for the compression achieved with $\phi_{0}=\left[-90^{\circ},-100^{\circ}\right]$, indicating that the

TABLE II. Bunch lengths (rms) of subbunches in a $4 \times 20 \mathrm{pC}$ bunch train with different injected phases $\phi_{0}$.

\begin{tabular}{lcccc}
\hline \hline$\phi_{0}(\mathrm{deg})$ & $\sigma_{z}^{1}(\mathrm{fs})$ & $\sigma_{z}^{2}(\mathrm{fs})$ & $\sigma_{z}^{3}(\mathrm{fs})$ & $\sigma_{z}^{4}(\mathrm{fs})$ \\
\hline 0 & 424 & 415 & 422 & 443 \\
-10 & 411 & 405 & 413 & 434 \\
-20 & 404 & 400 & 409 & 430 \\
-30 & 401 & 398 & 407 & 429 \\
-40 & 401 & 399 & 409 & 429 \\
-50 & 404 & 403 & 412 & 430 \\
-90 & 360 & 330 & 289 & 247 \\
-100 & 216 & 161 & 142 & 288 \\
-140 & 256 & 185 & 129 & 131 \\
-150 & 167 & 163 & 170 & 161 \\
-160 & 207 & 215 & 213 & 193 \\
-170 & 245 & 264 & 263 & 239 \\
-180 & 289 & 324 & 338 & 319 \\
-190 & 339 & 402 & 436 & 413 \\
-200 & 382 & 470 & 497 & 388 \\
-210 & 356 & 455 & 444 & 282 \\
-220 & 302 & 291 & 288 & 305 \\
\hline \hline
\end{tabular}




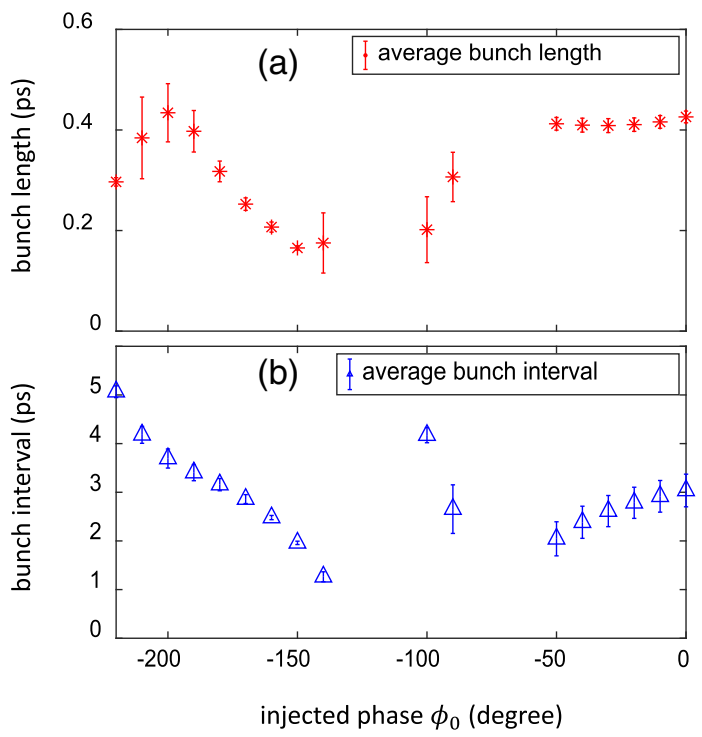

FIG. 10. Bunch lengths and bunch intervals with different injected phases $\phi_{0}$ for a $4 \times 20 \mathrm{pC}$ bunch train; (a) and (b) correspond to the statistics parameters in Tables II and III, respectively.

deep overcompression scheme is optimized for uniform compression of the bunch train.

The high-charge four-bunch case is shown in Figs. 11 and 12. The charge of each subbunch in the train is $200 \mathrm{pC}$, and the initial bunch current is $\sim 100$ A upon injection into the TWA tube. The initial UV laser interval is $8 \mathrm{ps}$ to generate separated bunches before the TWA tube, shown as the initial case in Fig. 11. It is still difficult to obtain a uniform bunch train when the injected phase is $\phi_{0}>-110^{\circ}$, as shown in the top and middle rows in

TABLE III. Bunch intervals in a $4 \times 20 \mathrm{pC}$ bunch train with different injected phases $\phi_{0}$.

\begin{tabular}{lccc}
\hline \hline$\phi_{0}(\mathrm{deg})$ & $t_{12}(\mathrm{ps})$ & $t_{23}(\mathrm{ps})$ & $t_{34}(\mathrm{ps})$ \\
\hline 0 & 3.38 & 3.02 & 2.71 \\
-10 & 3.25 & 2.90 & 2.60 \\
-20 & 3.11 & 2.77 & 2.47 \\
-30 & 2.94 & 2.60 & 2.30 \\
-40 & 2.72 & 2.37 & 2.06 \\
-50 & 2.40 & 2.03 & 1.70 \\
-90 & 3.11 & 2.73 & 2.12 \\
-100 & 4.00 & 4.30 & 4.22 \\
-140 & 1.38 & 1.22 & 1.19 \\
-150 & 2.00 & 1.94 & 1.94 \\
-160 & 2.45 & 2.46 & 2.53 \\
-170 & 2.78 & 2.84 & 2.96 \\
-180 & 3.04 & 3.14 & 3.29 \\
-190 & 3.25 & 3.38 & 3.58 \\
-200 & 3.51 & 3.67 & 3.90 \\
-210 & 4.03 & 4.14 & 4.37 \\
-220 & 4.94 & 5.08 & 5.18 \\
\hline \hline
\end{tabular}
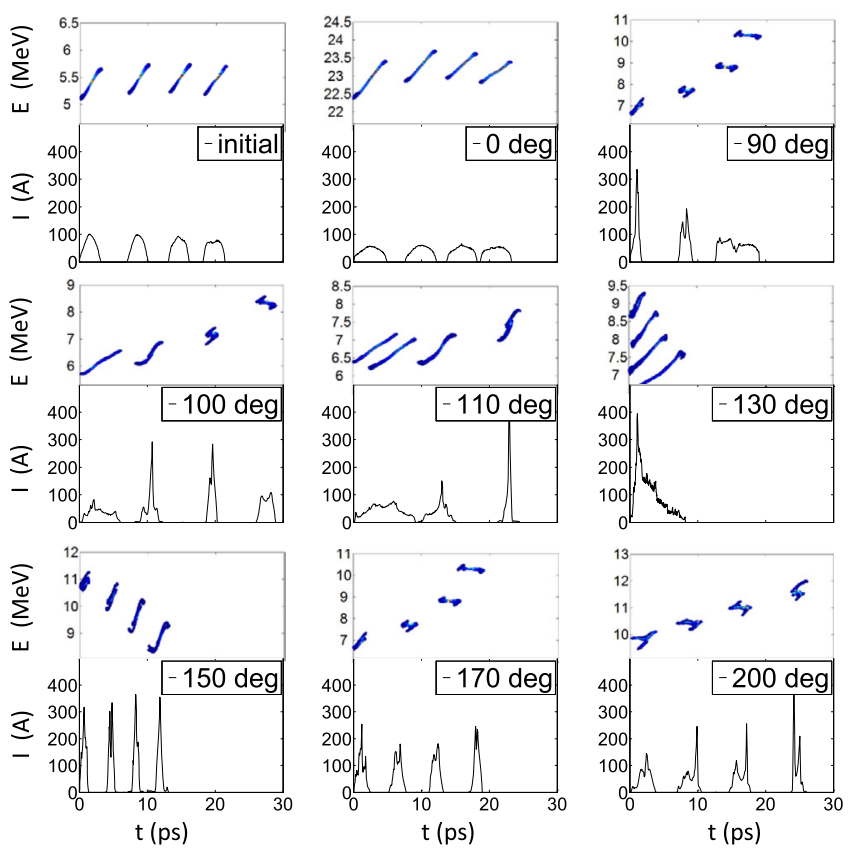

FIG. 11. High-charge four-bunch case: ASTRA simulations of the longitudinal distributions and phase spaces for the compression of a four-bunch train with different injected phases $\phi_{0}$, where the charge of each subbunch is $200 \mathrm{pC}$.

Fig. 11. However, when working in the deep overcompression mode $\left(\phi_{0}<-130^{\circ}\right)$, we obtain the typical optimized high-current four-bunch train distributions shown in the bottom row in Fig. 11. For the case of $\phi_{0}=-150^{\circ}$, the four-bunch distribution is relatively uniform in the time space, with a current of $\sim 300$ A for each compressed subbunch and a decreasing energy distribution.

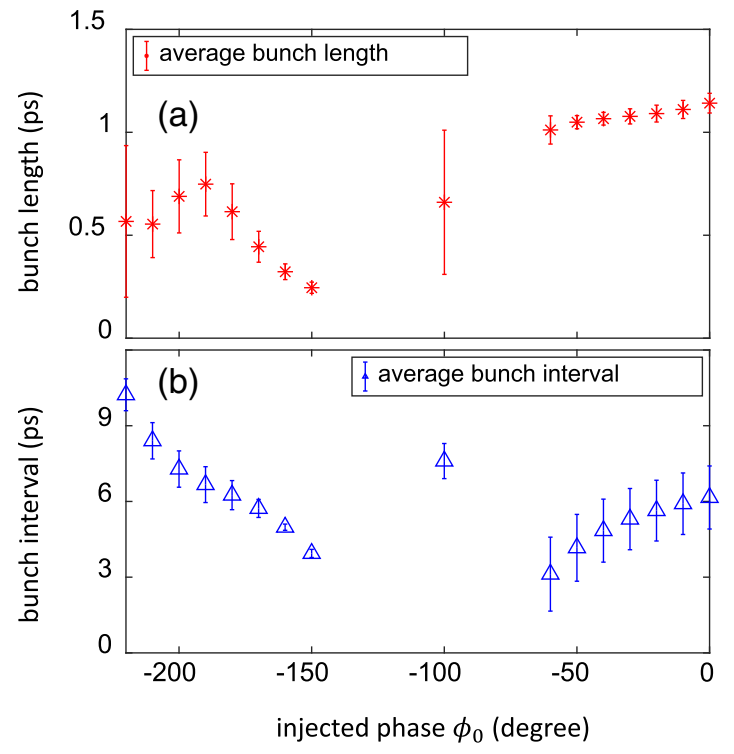

FIG. 12. Bunch lengths (a) and bunch intervals (b) with different injected phases $\phi_{0}$ for $4 \times 200 \mathrm{pC}$ bunch train. 
The average interval between adjacent subbunches is $\sim 4$ ps. For the case of $\phi_{0}=-170^{\circ}$, the four-bunch train is still relatively uniform, with an increased average interval of $\sim 6 \mathrm{ps}$, an increasing energy distribution, and a current of $\sim 200 \mathrm{~A}$ for each compressed subbunch. The four-bunch train distribution remains relatively uniformly spaced in time even when $\phi_{0}=-200^{\circ}$ (average interval of $\sim 7 \mathrm{ps}$ between subbunches). The parameter values are plotted (with error bars) versus the injected phase $\phi_{0}$ in Fig. 12. From this figure, we can see that, in the deep overcompression mode, the bunch lengths and intervals tend to be uniform (small error bars in Fig. 12) in this range, and the bunch intervals are continually tunable over a relatively wide range, which is beneficial for bunch-train compression.

\section{SUMMARY}

In summary, the deep overcompression mode of the velocity bunching scheme is greatly beneficial for bunchtrain compression due to its relatively large phase acceptance and its uniformity of compression. A theoretical model was built, and careful beam dynamics simulations based on ASTRA code were performed to study the deep overcompression scheme, in which the TWA tube reverses the phase space of the injected beam while maintaining a velocity difference (energy chirp) within the beam, thus giving rise to a compressed comblike electron bunch train after a few-meter-long downstream drift segment. The relatively uniform laser-comb bunch trains (both twobunch trains and four-bunch trains) that are generated via this optimized compression scheme, with charges of several hundred picocoulombs, subpicosecond subbunch lengths, and wide tunability in time and energy space, should have great potential for application in scenarios involving FELs, advanced particle acceleration, and terahertz radiation sources.

\section{ACKNOWLEDGMENTS}

This work was supported by the National Natural Science Foundation of China (NSFC Grant No. 11475097) and the National Key Scientific Instrument and Equipment Development Project of China (Grant No. 2013YQ12034504).

[1] A. Gover, Superradiant and stimulated-superradiant emission in prebunched electron-beam radiators. I. Formulation, Phys. Rev. ST Accel. Beams 8, 030701 (2005).

[2] M. Boscolo, M. Ferrario, I. Boscolo, F. Castelli, and S. Cialdi, Generation of short $\mathrm{THz}$ bunch trains in a $\mathrm{RF}$ photoinjector, Nucl. Instrum. Methods Phys. Res., Sect. A 577, 409 (2007).

[3] P. Muggli, V. Yakimenko, M. Babzien, E. Kallos, and K. P. Kusche, Generation of Trains of Electron Microbunches with Adjustable Subpicosecond Spacing, Phys. Rev. Lett. 101, 054801 (2008).

[4] S. Antipov, M. Babzien, C. Jing, M. Fedurin, W. Gai, A. Kanareykin, K. Kusche, V. Yakimenko, and A. Zholents, Subpicosecond Bunch Train Production for a Tunable mJ Level THz Source, Phys. Rev. Lett. 111, 134802 (2013).

[5] C. Jing, A. Kanareykin, J. Power, M. Conde, Z. Yusof, P. Schoessow, and W. Gai, Observation of Enhanced Transformer Ratio in Collinear Wakefield Acceleration, Phys. Rev. Lett. 98, 144801 (2007).

[6] P. Musumeci, R. Li, and A. Marinelli, Nonlinear Longitudinal Space Charge Oscillations in Relativistic Electron Beams, Phys. Rev. Lett. 106, 184801 (2011).

[7] Y. Shen, X. Yang, G. Carr, Y. Hidaka, J. B. Murphy, and X. Wang, Tunable Few-Cycle and Multicycle Coherent Terahertz Radiation from Relativistic Electrons, Phys. Rev. Lett. 107, 204801 (2011).

[8] Z. Zhang, Y. Ding, A. Marinelli, and Z. Huang, Longitudinal dynamics of twin electron bunches in the Linac Coherent Light Source, Phys. Rev. ST Accel. Beams 18, 030702 (2015).

[9] V. Petrillo et al., Dual color $\mathrm{x}$ rays from Thomson or Compton sources, Phys. Rev. ST Accel. Beams 17, 020706 (2014).

[10] P. Emma et al., First lasing and operation of an ångstromwavelength free-electron laser, Nat. Photonics 4, 641 (2010).

[11] T. Ishikawa et al., A compact X-ray free-electron laser emitting in the sub-ångström region, Nat. Photonics 6, 540 (2012).

[12] A. Lutman, R. Coffee, Y. Ding, Z. Huang, J. Krzywinski, T. Maxwell, M. Messerschmidt, and H.-D. Nuhn, Experimental Demonstration of Femtosecond Two-Color X-Ray Free-Electron Lasers, Phys. Rev. Lett. 110, 134801 (2013).

[13] G. De Ninno, B. Mahieu, E. Allaria, L. Giannessi, and S. Spampinati, Chirped Seeded Free-Electron Lasers: Self-Standing Light Sources for Two-Color Pump-Probe Experiments, Phys. Rev. Lett. 110, 064801 (2013).

[14] V. Petrillo et al., Observation of Time-Domain Modulation of Free-Electron-Laser Pulses by Multipeaked ElectronEnergy Spectrum, Phys. Rev. Lett. 111, 114802 (2013).

[15] T. Hara et al., Two-colour hard X-ray free-electron laser with wide tunability, Nat. Commun. 4, 2919 (2013).

[16] A. Marinelli, A. Lutman, J. Wu, Y. Ding, J. Krzywinski, H.-D. Nuhn, Y. Feng, R. Coffee, and C. Pellegrini, Multicolor Operation and Spectral Control in a GainModulated X-Ray Free-Electron Laser, Phys. Rev. Lett. 111, 134801 (2013).

[17] C. Ronsivalle et al., Large-bandwidth two-color freeelectron laser driven by a comb-like electron beam, New J. Phys. 16, 033018 (2014).

[18] A. Marinelli et al., High-intensity double-pulse X-ray freeelectron laser, Nat. Commun. 6, 6369 (2015).

[19] P. Chen, J. Dawson, R. W. Huff, and T. Katsouleas, Acceleration of Electrons by the Interaction of a Bunched Electron Beam with a Plasma, Phys. Rev. Lett. 54, 693 (1985).

[20] M. Litos et al., High-efficiency acceleration of an electron beam in a plasma wakefield accelerator, Nature (London) 515, 92 (2014). 
[21] Z. Zhang et al., Tunable High-Intensity Electron Bunch Train Production Based on Nonlinear Longitudinal Space Charge Oscillation, Phys. Rev. Lett. 116, 184801 (2016).

[22] G. L. Carr, M. C. Martin, W. R. McKinney, K. Jordan, G. R. Neil, and G. P. Williams, High-power terahertz radiation from relativistic electrons, Nature (London) 420, 153 (2002).

[23] C. Schroeder, C. Benedetti, E. Esarey, F. Grüner, and W. Leemans, Growth and Phase Velocity of Self-Modulated Beam-Driven Plasma Waves, Phys. Rev. Lett. 107, 145002 (2011).

[24] H. Zhang, I. Konoplev, A. Lancaster, H. Harrison, G. Doucas, A. Aryshev, M. Shevelev, N. Terunuma, and J. Urakawa, Non-destructive measurement and monitoring of separation of charged particle micro-bunches, Appl. Phys. Lett. 111, 043505 (2017).

[25] R. Pompili et al., First single-shot and non-intercepting longitudinal bunch diagnostics for comb-like beam by means of Electro-Optic Sampling, Nucl. Instrum. Methods Phys. Res., Sect. A 740, 216 (2014).

[26] B. Jiang, J. G. Power, R. Lindberg, W. Liu, and W. Gai, Emittance-Exchange-Based High Harmonic Generation Scheme for a Short-Wavelength Free Electron Laser, Phys. Rev. Lett. 106, 114801 (2011).

[27] Y. Sun, P. Piot, A. Johnson, A. Lumpkin, T. Maxwell, J. Ruan, and R. Thurman-Keup, Tunable Subpicosecond Electron-Bunch-Train Generation Using a TransverseTo-Longitudinal Phase-Space Exchange Technique, Phys. Rev. Lett. 105, 234801 (2010).

[28] S. Antipov, C. Jing, M. Fedurin, W. Gai, A. Kanareykin, K. Kusche, P. Schoessow, V. Yakimenko, and A. Zholents, Experimental Observation of Energy Modulation in Electron Beams Passing through Terahertz Dielectric Wakefield Structures, Phys. Rev. Lett. 108, 144801 (2012).
[29] M. Boscolo, M. Ferrario, C. Vaccarezza, I. Boscolo, F. Castelli, and S. Cialdi, in Proceedings of the 10th European Particle Accelerator Conference, Edinburgh, Scotland, 2006 (EPS-AG, Edinburgh, Scotland, 2006), p. 98.

[30] M. Ferrario et al., Experimental Demonstration of Emittance Compensation with Velocity Bunching, Phys. Rev. Lett. 104, 054801 (2010).

[31] M. Ferrario et al., Laser comb with velocity bunching: Preliminary results at SPARC, Nucl. Instrum. Methods Phys. Res., Sect. A 637, S43 (2011).

[32] B. Marchetti, M. Boscolo, M. Castellano, E. Chiadroni, M. Ferrario, B. Spataro, C. Vaccarezza, A. Cianchi, and I. T. Vergata, in Proceedings of the 2nd International Particle Accelerator Conference, San Sebastián, Spain (EPS-AG, Spain, 2011), THPS102, pp. 3672-3674.

[33] R. Pompili et al., Beam manipulation with velocity bunching for PWFA applications, Nucl. Instrum. Methods Phys. Res., Sect. A 829, 17 (2016).

[34] C. Tang, W. Huang, R. Li, Y. Du, L. Yan, J. Shi, Q. Du, P. Yu, H. Chen, T. Du, C. Cheng, and Y. Lin, Tsinghua Thomson scattering X-ray source, Nucl. Instrum. Methods Phys. Res., Sect. A 608, S70 (2009).

[35] K. Floettmann, http://www.desy.de.

[36] T. Shintake and M. Ferrario, High Performance Electron Injectors, Reviews of Accelerator Science and Technology (World scientific, 2010), Chap. 12, pp. 221-235.

[37] L. Serafini and M. Ferrario, Velocity bunching in photo-injectors, AIP Conf. Ser. 581, 87 (2001).

[38] D. Wang, L. Yan, Y. Du, J. Hua, Q. Du, H. Qian, X. Lu, W. Huang, H. Chen, and C. Tang, Generating ultrabroadband terahertz radiation based on the under-compression mode of velocity bunching, Rev. Sci. Instrum. 84, 022704 (2013). 